\title{
Travailler le jeu d'acteur avec Gilles Deleuze
}

\section{Flore Garcin-Marrou}

\section{OpenEdition}

Journals

Édition électronique

URL : https://journals.openedition.org/edl/3198

DOI : 10.4000/edl.3198

ISSN : 2296-5084

\section{Éditeur}

Université de Lausanne

\section{Édition imprimée}

Date de publication : 24 septembre 2020

Pagination : 173-192

ISBN : 978-2-940331-74-1

ISSN : 0014-2026

\section{Référence électronique}

Flore Garcin-Marrou, «Travailler le jeu d'acteur avec Gilles Deleuze », Études de lettres [En ligne], 313 | 2020, mis en ligne le 24 septembre 2021, consulté le 25 septembre 2021. URL : http:// journals.openedition.org/edl/3198; DOI : https://doi.org/10.4000/edl.3198 


\section{TRAVAILLER LE JEU D’ACTEUR AVEC GILLES DELEUZE}

Peut-on travailler le jeu d'acteur avec Gilles Deleuze? Sa philosophie est lue et étudiée dans les cours de théâtre. Mais trouve-t-on dans les œuvres complètes l'élaboration d'une théorie de l'acteur à proprement parler, susceptible d'inspirer une pédagogie, des pratiques de jeu à expérimenter? Nous proposons ici trois hypothèses de jeu, dans le sillage de la pensée deleuzienne: celle de l'acteur-mime, celle de l'acteur de la cruauté et celle de la machine actoriale.

Depuis sa petite enfance, "Gilles Deleuze n’a jamais cessé d'admirer le jeu des acteurs" nous confirme sa veuve Fanny Deleuze lors d'un entretien en $2011^{1}$. Il a été fasciné par la diction de Saturnin Fabre ${ }^{2}$, impressionné par Charles Dullin lorsqu'il le découvre dans le rôle de Jupiter, lors d'une représentation des Mouches de Jean-Paul Sartre à laquelle il assiste avec Michel Tournier en juin 1943, au Théâtre Sarah-Bernhardt ${ }^{3}$. Il a aimé la profonde voix d'Alain Cuny ${ }^{4}$, celui-là même qui a enregistré à la radio Pour en finir avec le jugement de dieu d'Antonin Artaud en 1947. Autour de 1968, quand Deleuze a entendu parler des happenings de Jean-Jacques Lebel ou du Living Theatre, il s'est mis à penser conjointement l'acteur et le concept d'événement, en portant son attention au stoïcisme et au théâtre d'Artaud dans Logique du sens. Plus tard, c'est

I. F. Deleuze, entretien avec l'auteur, 21/01/2011.

2. H. Guibert, «Portrait du philosophe en spectateur», entretien avec G. Deleuze, Le Monde, 06/10/1983, repris dans G. Deleuze, Deux régimes de fous, p. 197-203.

3. M. Tournier, Célébrations, p. 343.

4. G. Deleuze, Deux régimes de fous, p. 303 sq. 
vers des formes d'acting toujours plus radicales que Deleuze s'est tourné, comme la poésie sonore de Ghérasim Luca dont il commente à de nombreuses reprises les performances, ou bien la "machine actoriale ${ }^{5}$ de l'acteur et metteur en scène italien Carmelo Bene, qu'il qualifie d'« opérateur d'intensité» ${ }^{6}$. Gilles Deleuze ne s'est pas contenté d'admirer les acteurs. Il a également "fait l'acteur" à plusieurs reprises. Que ce soit dans une saynète politique sur le procès des mutins de la prison de Nancy en 1972, écrite par la journaliste Michèle Manceaux et mise en scène par Ariane Mnouchkine, destinée à être jouée en tomber de rideau de la pièce 1793 à la Cartoucherie et dans les banlieues ouvrières ${ }^{7}$, ou au cinéma dans le film de Michèle Rosier George qui? (1973), lorsqu'il incarne le rôle du prêtre et philosophe chrétien Lamennais donnant la réplique à Anne Wiazemsky. La problématique qui nous occupe dans cet article est celle-ci: Deleuze a fait coïncider sa pensée avec la problématique du jeu d'acteur à plusieurs reprises. En identifiant ces coïncidences, peut-on extraire du discours philosophique des pistes concrètes utiles pour un travail de recherche scénique? Le fait est que cette philosophie est lue et étudiée dans les cours de théâtre. Mais sur quelles bases philosophiques pourrait-on travailler le jeu d'acteur avec Gilles Deleuze? Nous proposons ici, dans un geste de réappropriation et de traduction scénique de la pensée de Deleuze, trois hypothèses de techniques de jeu à expérimenter dans un laboratoire d'acteurs: celle de l'acteur-mime, celle de l'acteur de la cruauté et celle de la machine actoriale.

5. G. Deleuze, «Carmelo Bene», p. 111 sq.

6. Ibid.

7. Lors du procès, six prisonniers sont inculpés après un mouvement pour la reconnaissance de leurs droits communs. Ils reçoivent l'appui immédiat du GIP (Groupe d'information sur les prisons): Gilles Deleuze est cité comme témoin par la défense. Manceaux et Mnouchkine font une retranscription des débats, publiée dans la revue Esprit. Lors de ces représentations, Deleuze joue non pas son rôle, mais celui d'un policier encadrant les accusés, en compagnie de Daniel Defert, Michel Foucault et Philippe Meyer. Le texte de la pièce a été reproduit dans Ph. Artières et al. (dir.), Le groupe d'information sur les prisons, p. 237-257; publié originellement dans la revue Esprit, octobre 1972, p. 524-555. 


\section{Première hypothèse de jeu: l'acteur-mime}

Dès Différence et répétition (1953), Deleuze affirme que «les reflets, les échos, les doubles [...] ne sont pas du domaine de la ressemblance, de l'équivalence» ${ }^{8}$. Autrement dit, ce qui est répété n'est pas remplacé par de l'identique, mais par une variation, une altération. La répétition est

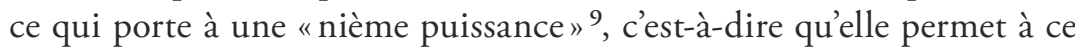
qui est répété de gagner en puissance et de se différencier. C'est le principe du "par cœur» chez le comédien: il répète son texte, non pour en livrer une interprétation identique à chaque représentation, mais pour en éprouver les puissances et les forces cachées, sans cesse renouvelées. Le texte se trouve gonflé d'une intensité nouvelle. La répétition fait surgir des puissances révélées selon un processus de métamorphose et de différenciation. Ce type de répétition qui crée de la différence n’a rien à voir, explique Deleuze, avec "l'effort de l'acteur qui "répète" dans la mesure où la pièce n'est pas encore sue ${ }^{10}$. La répétition qui sert à la mémorisation est perçue comme une répétition du même.

Mais outre l'analyse du "par cœur» chez le comédien, c'est une réflexion récurrente sur l'art du mime et la singularité de celui que nous nommerons à partir de maintenant l'acteur-mime qui guide Deleuze dans ses investigations sur le concept de répétition. Nous savons grâce à Fanny Deleuze que la performance de Jean-Louis Barrault en Pierrot dans Les Enfants du Paradis de Marcel Carné l' "écœura" ${ }^{11}$. Pour quelles raisons? Considérait-il la performance de Barrault du mime Deburau interprétant Pierrot comme une exemplification de la répétition mimétique, c'est-à-dire de la répétition du même? Nous ne le saurons jamais. Ce qui intéresse profondément Deleuze lorsqu'il pense le mime, dans la spécificité de cette pratique artistique, c'est justement qu'il n'est jamais strictement mimétique. En effet, «l'imitation la plus simple comprend la différence " ${ }^{12}$. Pour se figurer concrètement ce que Deleuze veut dire par là, il suffit de regarder un cours du mime Marceau pour comprendre que chaque geste mimé n'est pas motivé par l'imitation exacte de la nature,

\footnotetext{
8. G. Deleuze, Différence et répétition, p. 7.

9. Ibid., p. 8.

Io. Ibid., p. 19.

II. F. Deleuze, entretien avec l'auteur, 21/01/2011.

I2. G. Deleuze, Différence et répétition, p. 35.
} 
mais par la stylisation et la rythmicité d'un geste acquérant un changement d'amplitude et de qualité. Par exemple, la marche est travaillée par Marceau dans son immobilité afin qu'elle soit plus lisible pour le spectateur. Il faut que le geste soit travaillé différemment que dans la réalité. Pour le mime, il suffit qu'un signe traduise une idée, procède à une traduction de l'hétérogénéité. Celui qui mime émet un signe différent (traduit, stylisé, rythmé, épuré) de ce qui est pris pour modèle. Le résultat de l'imitation n'appartient pas à la même réalité que le modèle. Le geste de traduction est exécuté grâce à l'impulsion d'une autre idée, d'un autre mouvement, et en ceci, introduit de la différence. Deleuze compare le mime avec la relation instaurée entre le pédagogue et l'apprenant:

Nous n'apprenons rien avec celui qui nous dit: fais comme moi. Nos seuls maîtres sont ceux qui nous disent "fais avec moi", et qui, au lieu de nous proposer des gestes à reproduire, [savent] émettre des signes à développer dans l'hétérogène ${ }^{13}$.

Mimer revient à comprendre l'Autre, faire émerger la différence dans l'espace répétitif.

Dans Logique du sens, Deleuze fait référence au texte de Mallarmé "Mimique» (1886) qui relate une pantomime écrite et jouée par Paul Margueritte: Pierrot assassin de sa femme. L'intrigue est simple: Pierrot raconte de quelle manière il a tué Colombine, après qu'elle l'a trompé. Après l'avoir liée au lit, il la chatouille jusqu'à la mort. Pierrot entreprend de mimer la scène en endossant son propre rôle et celui de Colombine. Pendant cette simulation alternative (une forme de "vice-diction» écrit Deleuze), l'ombre de Colombine réapparaît alors que Pierrot se tord réellement de douleur sous sa propre torture. Il tombe mort sur le portrait de sa femme. Cette mise en abyme de l'imitation, cette imitation d'imitation (deux performances mimées sont ici enchâssées, celle d'un acteur mimant son rôle de Pierrot, celle du mime Pierrot se mimant lui-même en train de commettre l'irréparable) suscite un commentaire singulier de Mallarmé:

La scène n'illustre que l'idée, pas une action effective, dans un hymen (d'où procède le Rêve), vicieux et sacré, entre le désir et l'accomplissement, la perpétration et son souvenir: ici devançant, là remémorant,

13. Ibid. 
au futur, au passé, sous une apparence fausse de présent. Tel opère le Mime, dont le jeu se borne à une allusion perpétuelle sans briser la glace: il installe, ainsi, un milieu, pur, de fiction ${ }^{14}$.

Le dispositif enchâssé décrit par Mallarmé (un mime mimant un personnage en train de mimer) met le mime dans une disposition de ne pas imiter une action, mais plutôt d'imiter le processus d'imitation en lui-même. Pour le dire autrement, Pierrot ne cherche pas à mimer une action qui aurait déjà été accomplie. Ce qui intéresse différemment le mime mimant Pierrot se mimant en train d'assassiner Colombine, c'est le processus qui relie l'action passée et le présent de l'action en train de se faire. Mallarmé décrit ce dispositif temporel spécifique où le présent devient une virtualité, et le personnage une sorte de fantôme qui donne à voir une page blanche en train de s'écrire, un mouvement en train d'être effectué. Pour Mallarmé, le mime est une voie d'exploration d'un nouveau théâtre qui ne relève pas de l'action aristotélicienne, mais plutôt de l'effectuation du mouvement. Pour Deleuze, le mime mallarméen est "celui qui contre-effectue l'événement", c'est-à-dire qu'il ne cherche pas à "imiter le vécu" ${ }^{15}$, ni à reproduire un état de choses, ni à donner à voir une image. L'action n'est pas dramatique au sens classique, ni directement référentielle. Au contraire, ce que le mime mallarméen arrive à faire, c'est d'extraire l'action (drama) de sa fonctionnalité narrative pour faire surgir une "action" d'une autre qualité, d'une autre nature, que Deleuze nomme "événement». Le philosophe, notamment dans Logique $d u$ sens $^{16}$, montre à l'exemple du mime comment l'acteur peut sortir du registre mimétique pour devenir, non plus un personnage de la fable qu'il raconte, mais un agent de l'événement scénique. Lorsque le mime Marceau fait travailler à ses élèves la montée d'un escalier, il n'insiste pas sur la capacité représentative du geste (le geste comme une composante de la fable), mais davantage sur sa qualité intensive et son aptitude à créer la pesanteur du lieu, à faire ressentir la résistance de la rampe opposée au corps qui s’y agrippe, ou l'effort fourni par le corps gravissant

I4. S. Mallarmé, "Mimique», in Oeuvres complètes, vol. 2, p. 178 sq.

I5. G. Deleuze, G. Guattari, Qu'est-ce que la philosophie?, n. 3, p. 151.

I6. G. Deleuze, Logique du sens, p. 80, 160, 173, 188, 256, 297 sq., 303, 325, 327, 332 , 348. Le mime mallarméen qui subvertit la mimèsis est également analysé par Jacques Derrida dans «La double séance», p. 253, 258. Le texte est d'abord paru dans Tel Quel, 41-42 (1970), un an après la publication de Logique du sens. 
les marches. Le spectateur est alors confronté à une autre temporalité que le temps dramatique structuré par un début et une fin: le temps de l'événement.

Ce temps propre, Deleuze l'identifie comme le temps de l'Aiôn ${ }^{17}$. Ce temps indéterminé, ou cette extra-temporalité contenue dans l'immanence du présent, se manifeste selon Deleuze à la surface des corps comme une virtualité. Le mime est ainsi celui qui effectue l'événement, dans toute sa processualité:

L'événement, c'est que jamais personne ne meurt, mais vient toujours de mourir et va toujours mourir, dans le présent vide de l'Aiôn, éternité ${ }^{18}$.

À cette nouvelle forme de temps (l'Aiôn) correspondrait une autre manière de jouer. Laction dramatique de type classique, qui donne une interprétation du monde suivant les règles d'une apparente unité, s'efface devant une dramaturgie du coup de dés dont l'acteur est le premier producteur. Un type d'acteur que Deleuze désigne, dans Différence et répétition, comme un joueur:

Le joueur s'abandonne temporairement à la vie, et temporairement fixe son regard sur elle; l'artiste se place temporairement dans son œuvre, et temporairement au-dessus de son œuvre; l'enfant joue, se retire du jeu et y revient. Or ce jeu du devenir; c'est aussi bien l'être du devenir qui le joue avec lui-même: l'Aiôn, dit Héraclite, est un enfant qui joue, qui joue au palet ${ }^{19}$.

Ce théâtre de l'événement se joue toujours à l'infinitif: l'action n'a jamais fini de s'accomplir. L'acteur n'est pas un support de la représentation, mais un agent du devenir, c'est-à-dire d'un processus qui lui permet d'incarner un événement. Cette forme de jeu dans le vif, existant pour elle-même indépendamment de la fable ou d'un texte, est particulièrement reconnaissable dans le domaine de la performance. L'Aiôn est le temps suspendu que le performeur arrive à créer entre passé et futur: ce

17. Dans la philosophie antique, l'Aiôn (pouvant être traduit par l'éternité, un long espace de temps indéterminé) est un des trois principaux concepts du temps, avec Chronos (le temps linéaire) et Kairos (le temps opportun, l'occasion).

I8. G. Deleuze, Logique du sens, p. 80.

19. G. Deleuze, Différence et répétition, p. 28. 
milieu, dont parle Deleuze, où se déploie intensivement un geste. Nous pensons par exemple à la performance de Marina Abramovic, Balkan Baroque (1997). La performeuse nettoie méthodiquement avec une brosse en métal des os ensanglantés empilés à côté d'elle. La performance dure six heures par jour pendant quatre jours d'affilée. Outre que l'action dénonce les massacres perpétrés pendant la guerre en Yougoslavie, c'est la répétition du geste qui n'en finit pas d'être répété pendant quatre longs jours qui constitue le cœur de l'événement performatif.

L'hypothèse de l'acteur-mime est approfondie dans Logique du sens par l'évocation de la figure de l'acteur stoïcien. Sur le plan de la connaissance, les stoïciens s'attachent à une théorie de la surface où se manifestent des événements plutôt que des vérités fixes et immuables. Cette surface est un lieu de ce qui advient et non de ce qui est. Il s'y manifeste ce qui n'est pas fixé par une qualité mais ce qui est sans cesse repris dans des processus - tout à fait comme le personnage d'Alice, paradigme carrollien de l'acteur stoïcien, qui grandit, rapetisse et se métamorphose sans cesse. Tout ce qui lui arrive semble être le fruit d'un coup de dé. Elle glisse à la surface de la narration, rebondit, se condense, change d'état et d'échelle. Elle n'est déterminée que par les actions qu'elle accomplit au gré du dynamisme qui l'entraîne coûte que coûte: ce qui se joue pour Alice est au-delà des significations. Elle se déplace, arpente, crée une nouvelle géographie de la scène qui s'agence par coup de dés, assumant un autre type de sens que Carroll nomme le nonsense: «le sens apparaît et se joue à la surface» précise Deleuze ${ }^{20}$.

Théâtre pour de brusques condensations, fusions, changements d'états des couches étalées, distributions et remaniements de singularités, la surface peut s'accroître indéfiniment, comme lorsque deux liquides se dissolvent l'un dans l'autre. Il y a donc toute une physique des surfaces $[\ldots]$ qui recueille sans cesse les variations ${ }^{21}$.

Procédant par analogie, peut-être faudrait-il aller chercher du côté du théâtre de Valère Novarina pour trouver un point de comparaison avec Alice. Évoquons notamment la performance d'André Marcon dans Le Monologue d'Adramélech mis en scène par Christian Rist, créé en 1984.

20. G. Deleuze, Logique du sens, p. 158.

2I. Ibid., p. 150. 
Annie Gay, dans la revue Théatre/public (1985), décrit l'acteur en ses termes:

Il entre, la tête levée, en état d'apesanteur. Il danse un pas qui ne se poserait pas. Il danse une marche sur les eaux, il lévite un peu comme si, à l'intérieur du costume, les muscles avaient disparu, comme si s'était évanouie l'infime présence électrique nécessaire à leur tonus. Puis il se pose doucement ${ }^{22}$.

Et il se met à parler au public. Il apparaît, pour Annie Gay, comme la figure de "l'acteur novarinien», l'acteur «du drame de la vie» qui vide son corps pour accueillir le texte dans un moment de plaquage au sol d'un corps des surfaces:

André Marcon touche [...] l'ultime palier de son intensité dans une danse accélérée, tournoyante et terrible, entravée par le poids du manteau dont l'étoffe lourde [...] semble rabattre au sol son impossible envol échappatoire ${ }^{23}$.

Les autres personnages de Valère Novarina semblent eux aussi glisser sur la scène, glisser sur le sens, se tenir à la surface du langage, «le long du rideau " ${ }^{24}$. Ce qui est donné à voir et à entendre ne relève pas de la fable aristotélicienne: c'est autre chose que le théâtre de Novarina raconte. Une nouvelle logique scénique: celle des métamorphoses du langage, des corps, de la parole, celle du non-sens où les acteurs sont des «[animaux plats] des surfaces» ${ }^{25}$.

Lacteur des surfaces travaille dans le sens d'une suspension, entre devenir et histoire, dans un refus de se résigner à ce qui advient. Chaque événement est double: l'acteur reste dans l'instant alors que le personnage qu'il joue espère quelque chose de l'avenir ou se remémore son passé. Lacteur fait alors éprouver cette double tension entre la virtualité de son rôle et l'actualité de sa présence sur scène. Tel est l'art de l'acteur: limiter l'effectuation de l'événement à un présent sans mélange, rendre l'instant d'autant plus intense qu'il exprime un futur et un passé

22. A. Gay, «L'acteur délié», p. 13 sq.

23. Ibid.

24. "Et s'il n'y a rien à voir derrière le rideau, c'est que tout le visible, ou plutôt toute la science possible est le long du rideau»(G. Deleuze, Logique du sens, p. 19).

25. Ibid. 
illimités. Il n'y a aucune transcendance ici. L'acteur n'est pas saisi par une transe. Le surgissement intensif de l'événement se fait sur un plan d'immanence: l'acteur devient alors "digne de ce qui [lui] arrive» ${ }^{26}$, fait surgir l'événement et échappe à la fable dramatique et à la représentation mimétique. Ce dédoublement de l'acteur, dans le temps illimité de l'événement, amène Deleuze à commenter dans la vingt-et-unième série de Logique du sens, le paradoxe de Diderot en utilisant ces mots:

L'acteur n'est pas comme un dieu, plutôt comme un contre-dieu. Dieu et l'acteur s'opposent par leur lecture du temps. Ce que les hommes saisissent comme passé ou futur, le dieu le vit dans son éternel présent. Le dieu est Chronos: le présent divin est le cercle tout entier, tandis que le passé et le futur sont des dimensions relatives à tel ou tel segment qui laisse le reste hors de lui ${ }^{27}$.

Deleuze réfute l'idée que l'acteur acquiert une conscience infinie et puisse faire événement en occupant une parcelle d'éternité:

Au contraire, le présent de l'acteur est le plus étroit, le plus resserré, le plus instantané, le plus ponctuel, point sur une ligne droite qui ne cesse de diviser la ligne, et de se diviser lui-même en passé-futur. L'acteur est de l'Aiôn: au lieu du plus profond, du plus plein présent, présent qui fait tache d'huile, et qui comprend le futur et le passé, voici surgir un passé-futur illimité qui se réfléchit en un présent vide n'ayant pas plus d'épaisseur que la glace. L'acteur représente, mais ce qu'il représente est toujours encore futur et déjà passé, tandis que sa représentation est impassible, et se divise, se dédouble sans se rompre, sans agir ni pâtir ${ }^{28}$.

La capacité de l'acteur à se dédoubler, entre le temps lié à sa chair et le temps de son surgissement sur la surface de la scène, forme un paradoxe essentiel :

C'est en ce sens qu'il y a un paradoxe du comédien: il reste dans l'instant, pour jouer quelque chose qui ne cesse de devancer et de retarder, d'espérer et de rappeler ${ }^{29}$.

26. Ibid., p. 175.

27. Ibid., p. 176.

28. Ibid.

29. Ibid. 


\section{Deuxième hypothèse: l'acteur de la cruauté}

À l'acteur des surfaces succède, dans Logique du sens, l'évocation d'un autre type d'acteur: l'acteur des profondeurs, identifié comme l'acteur de la cruauté, participant d'un système dramaturgique que Deleuze reconnaît d'abord dans le théâtre d'Eschyle qui "énonce les rapports finis du corps existant avec des forces qui l'affectent " ${ }^{30}$, en l'opposant au théâtre de Sophocle qui lui, participe du théâtre de la représentation mimétique et du jugement, condamnant ses personnages à un asservissement sans fin au destin. Quelles sont les qualités de l'acteur de la cruauté? S'il ne se définit pas par sa capacité à imiter le monde, à reproduire des sentiments, il s'impose comme un corps qui, par l'intensité de sa performance, accède à une intensité de présence. Lors de sa lecture au Théâtre du Vieux-Colombier en 1947, le corps supplicié d'Antonin Artaud est devenu la scène elle-même. Artaud tient cette conception de l'acteur des cours reçus de Charles Dullin à l'Atelier. Dans un article de 1922, Artaud loue l'entreprise "d'assainissement " et de "régénération" de Dullin, qui met en place avec une équipe d'acteurs des «méthodes [de jeu] nouvelles instaurées». La méthode d'improvisation proposée par Dullin à ses acteurs "force l'acteur à penser ses mouvements d'âme au lieu de les figurer». Il ne s'agit plus d'imiter les sentiments, mais de les vivre. Celui qui réussit cela est qualifié d'acteur «idéal» ${ }^{31}$. Dans la pratique actoriale de la cruauté, le corps ne figure plus une action, mais vibre, tremble, crie, éructe et met le spectateur en contact avec des forces vives. Dans le théâtre de la cruauté, «il y a donc bien des acteurs et des sujets, mais ce sont des larves, parce qu'elles sont seules capables de supporter les tracés, les glissements et rotations " ${ }^{32}$. L'acteur de la cruauté est une "sorte d'élément passif et neutre " ${ }^{33}$ que Deleuze à son tour qualifiera de "sujet larvaire", "corps intensif" poussant des "cris" et articulant des «mots-souffles». C'est bien ce type de corps qui crache, crie, défèque dans l'émission radiophonique d'Artaud Pour en finir avec le jugement de dieu: "corps volcanique", "corps-théâtre atomique», corps

30. G. Deleuze, Critique et clinique, p. 161.

3I. A. Artaud, "L'atelier de Charles Dullin", revue Action, 1921/1922 (Euvres complètes, vol. 2, p. 134 sq.; (Euvres, p. 35).

32. G. Deleuze, Différence et répétition, p. 282 sq.

33. A. Artaud, "Le Théâtre de la cruauté. Premier manifeste», in Euvres, p. 564. 
réduit à un "gaz puant» ${ }^{34}$ ou encore "corps sans organe» - expression artaudienne que Deleuze consacrera comme un des piliers conceptuels de L'anti-CEdipe. Qu'est-ce qu'un corps sans organe? Alors que dans un organisme vivant, les organes sont "juges et jugés» d'après une organisation décidée par l'ordre naturel et commun à tous, le corps qui échappe au jugement est un corps "vital et vivant", sans organes, "affectif, intensif, anarchiste, qui ne comporte plus que pôles, des zones, des seuils et des gradients", traversé par une "vitalité non-organique» ${ }^{35}$. Le corps sans organe de l'acteur de la cruauté est passé «sur la table d'autopsie pour lui refaire son anatomie» parce que l'homme est mal construit et doit être "délivré de tous ses automatismes et rendu à sa véritable liberté» ${ }^{36}$.

Comment interagissent les corps des acteurs de la cruauté? Le système de la cruauté implique le combat (agôn) plutôt que le jugement (krisis): un combat entre des puissances prises dans des rapports de force. Chez Artaud, selon Deleuze, cela implique une nouvelle "posture des corps " ${ }^{37}$. Le combat n'est pas synonyme d'anéantissement. Au contraire, un corps soumis au combat gagne en vitalité, en volonté de puissance. Ce qu'Artaud développe dans son texte Le Théatre et la peste (où il soutient que la peste est une épidémie mortelle qui réactive paradoxalement les forces et la vitalité), Deleuze le réutilise pour construire sa théorie sur les personnages-machines de guerre dans Mille plateaux ${ }^{38}$ : des personnages qui ont la particularité de courir un grand danger et qui tracent une ligne de fuite, pleine expression de leur liberté qu'ils paient parfois de leur vie. L'acteur de la cruauté mène en effet un combat: l'implication de son corps lui fait mobiliser de nouvelles forces qu'il ne représente pas mais qu'il fait exister. L'espace scénique, qui n'est plus mimétique, devient un espace d'émergence de forces nouvelles. Dans ce "théâtre de forces", ce "théâtre vitaliste», ce théâtre préjudicatif (d'avant le jugement), l'acteur est une flèche décochée au hasard par la nature (comme le "philosophe-comète» ${ }^{39}$ nietzschéen). Il ne s'inscrit plus ni

34. A. Artaud, Pour en finir avec le jugement de dieu, p. 1650.

35. G. Deleuze, Critique et clinique, p. 164.

36. A. Artaud, Pour en finir avec le jugement de dieu, p. 1654.

37. G. Deleuze, Critique et clinique, p. 165.

38. G. Deleuze, G. Guattari, "Traité de nomadologie».

39. G. Deleuze, Nietzsche et la philosophie, p. 121. Cf. F. Nietzsche, «Schopenhauer éducateur» $\$ 7$, p. 76. 
dans le devenir historique ni dans le devenir scénique. Il n'est plus agent du drame comme le conçoit Aristote. L'acteur joue contre son temps, ni éternel, ni historique, mais intempestif et inactuel ${ }^{40}$. L'acteur de la cruauté est un "homme tropical» ${ }^{41}$ qui se distingue de l'acteur «moral, méthodique ou modéré " ${ }^{42}$. Il fuit les "zones tempérées " ${ }^{43}$ et vient côtoyer les lieux extrêmes à des heures extrêmes, "où vivent et se lèvent les vérités les plus hautes, les plus profondes " ${ }^{44}$.

Voici ce que pourrait être en théorie l'acteur du théâtre de la cruauté. Mais qui est-il en pratique? Nous l'avons identifié dans une mise en scène de 1923 de Georges Pitoëff des Six personnages en quête d'auteur de Pirandello. Lorsqu'en 1923, Antonin Artaud consigne ses impressions après avoir assisté à cette représentation, il décrit des acteurs maquillés de blanc aux allures fantomatiques. Ces entités larvaires apparaissent sur scène en réclamant à l'auteur d'être des personnages, c'est-à-dire d'accéder à la représentation. Ces entités larvaires et spectrales «en quête d'un moule où se couler " ${ }^{45}$ sont typiquement des acteurs de la cruauté. Selon Artaud, ces acteurs-larves portent d'"autres corps humains", vivant "dans les profondeurs / de certaines tombes / en des endroits historiquement / sinon géographiquement insoupçonnés » ${ }^{46}$. Le commentaire de Deleuze se place dans la continuité de cette évocation. L'acteur larvaire vit la dissolution de son moi : il ne se définit plus par l'unité de sa subjectivité mais est constitué de "contractions", de "contemplations", de "fatigues", de "présents variables» ${ }^{47}$, participant à un théâtre de signes qui échappe à la parole articulée, exprimé par des «gestes et des attitudes ayant une valeur idéographique» ${ }^{48}$.

40. G. Deleuze, Nietzsche et la philosophie, p. 122.

4I. Ibid., p. 126.

42. Ibid.

43. Ibid.

44. Ibid.

45. A. Artaud, "Six personnages en quête d'auteur à la Comédie des Champs-Élysées", La Criée, 24 (mai 1923) (Euvres complètes, vol. 2, p. 142 sq.; CEuvres, p. 39 sq.).

46. A. Artaud, "Le théâtre de la cruauté», in Oeuvres, p. 1661.

47. G. Deleuze, Différence et répétition, p. 107. C'est ce qu'Antonin Artaud remarque à propos des corps des danseurs balinais, de "véritables hiéroglyphes", dont "les gestes au lieu de représenter les mots, des corps de phrases, [...] représentent des idées, des attitudes de l'esprit» (A. Artaud, La mise en scène métaphysique, in Euvres, p. 1935). 48. Ibid. 
Nous pouvons avancer une deuxième hypothèse sur ce que pourrait être en pratique l'acteur de la cruauté: il pourrait s'agir de l'acteur baroque, décrit par le metteur en scène de théâtre Eugène Green dans La parole baroque. L'acteur baroque a ceci de particulier qu'il est amené à opérer très peu de déplacements physiques sur scène. Son interprétation ne réside pas dans sa façon d'incarner le texte, mais dans la manière dont il va insuffler de l'énergie dans les mots, dont il va maîtriser son souffle. C'est le geste qui apporte une énergie visuelle à la parole. L'acteur baroque fonctionne comme une machine. Les passions sont le résultat de passions mécaniques. Le rôle est transmis sous forme d'énergie qui permet au corps de se mouvoir (et ce n'est donc pas l'intention psychologique qui commande le geste). Le corps baroque est un corps d'énergie, un "vide où passe l'énergie " ${ }^{49}$ : il n'a "pas de formes fixes ou closes, mais est conçu comme un lieu de passage et de mouvement perpétuel " ${ }^{50}$, il «n'est jamais vraiment en état statique, mais exprime toujours des tensions ${ }^{51}$ et "présente une harmonie qui naît non pas de la perfection de formes finies, mais d'un équilibre entre tensions opposées " 52 (le combat spécifique à l'acteur de la cruauté dont nous parlions plus haut). Ce corps baroque présente ainsi des caractéristiques similaires à l'acteur-larvaire, subissant constamment des mouvements de déformation, traduisant une énergie en devenir.

\section{Troisième hypothèse: la machine actoriale}

Les réflexions sur l'acteur prennent un nouvel aspect dans L'Anti-CEdipe qui marque un changement de paradigme dans la pensée deleuzienne du théâtre. L'inconscient n'est plus considéré comme un théâtre mais comme une usine, un lieu et un agent de production. Le théâtre devient «théâtre de la production" et le corps sans organes le paradigme du corps de l'acteur de la production. La référence vient d'Artaud qui, dans Van Gogh ou le suicidé de la société, indique que «le corps sous la peau est

49. E. Green, La parole baroque, p. 150.

50. Ibid.

5I. Ibid.

52. Ibid. 
une usine surchauffée " ${ }^{53}$. L'acteur est un "corps-machine ${ }^{54}$ connecté à d'autres machines, en interrelation avec la nature, un milieu, une atmosphère. Le corps de l'acteur est un corps schizophrénique, subissant la désorganisation de ses organes:

Le corps est le corps Il est seul Et n'a pas besoin d'organes Le corps n'est jamais un organisme. Les organismes sont les ennemis du corps. Le corps sans organes s'oppose moins aux organes qu'à cette organisation des organes que l'on appelle organisme. C'est un corps intense, intensif. Il est parcouru d'une onde qui trace dans le corps des niveaux ou des seuils d'après les variations de son amplitude. Le corps n'a donc pas d'organes, mais des seuils ou des niveaux ${ }^{55}$.

Comme le schizophrène vivant la désintégration de son moi, la machine actoriale propre à celui que nous nommerons «l'acteur-schizo" n'interprète pas de personnages fixés par une psychologie mais délire des séries de travestissements, véritable être de la métamorphose enchaînant de multiples masques ou de multiples intensités "dépouillées de leur figure et de leur forme " ${ }^{56}$. L'acteur-schizo a les mêmes caractéristiques que l'acteur larvaire, analysé plus tôt. Cette forme particulière de corps implique néanmoins une nouvelle forme de "dialogue» entre acteurs: le propre d'un corps-machine est d'être couplé à d'autres machines. Une machine produit un flux que l'autre reçoit en coupant le flux. Lors d'une conversation, ce n'est pas un échange entre deux individus déterminés par une psychologie qui opère, mais un échange transversal de flux, de connexions, de disjonctions au sein d'une multiplicité de possibilités ${ }^{57}$. Cette économie des flux de désir peut être transposée au théâtre dans une conception de l'interrelation non-psychologique entre les personnages d'une même pièce, telle que Meyerhold, par exemple, la conçoit, au cours de ses recherches sur le corps de l'acteur. Autrement dit, il s'agit d'une interrelation entre personnages sur le mode de l'action et de la réaction physiques. Le jeu de l'acteur s'opère via une biomécanique qui fait de l'artiste un ingénieur. L'acteur est tenu d'être réceptif aux vitesses,

53. A. Artaud, Van Gogh ou le suicidé de la société, p. 1459.

54. G. Deleuze, F. Guattari, L'Anti-CEdipe, p. 7.

55. G. Deleuze, Francis Bacon, p. 33.

56. G. Deleuze, F. Guattari, L'Anti-CEdipe, p. 26.

57. Ibid., p. 71. 
aux intensités et aux flux qui relient les personnages entre eux et traversent le sien. L'acteur envisage son personnage en termes d'effets. Le rôle est abordé selon ses rouages et le corps est traversé par des noms, des délires, des désirs. L'acteur ne se demande plus à propos de son rôle: "qu'est-ce que ça veut dire?» mais plutôt "comment ça marche?» ${ }^{58} \mathrm{Il}$ n'est pas dans une attention au sens, mais plutôt à la machinerie, au fonctionnement de son rôle qu'il aborde désormais comme une partition. Le personnage n'a plus un nom mais des noms: Hamlet n'est plus un seul personnage, mais une entité traversée par des intensités vibratoires. L'acteur n'est plus en position d'interpréter son personnage mais d'expérimenter son personnage, en même temps qu'il le joue. Il ne s'agit pas pour un acteur d'interpréter un texte en s'en tenant à sa signification, mais de le considérer comme une "machine littéraire» à laquelle son "corps-machine" 59 doit se coupler. Le metteur en scène tient alors davantage du «mécanicien ${ }^{60}$.

En pratique, Carmelo Bene matérialise cette conception machinique de l'acteur que Deleuze qualifie de «machine actoriale». Bene conçoit un acteur démiurge et artisan, devenant "opérateur d'intensités» ${ }^{61}$, acteur et metteur en scène à la fois. Le corps sans organes de l'acteur n'est plus que les décombres d'un corps désorganisé, traduisant l'impossibilité d'agir du personnage, son impossibilité de parler. Bene expérimente la dévastation du dire dans son spectacle Pinocchio: il bouleverse la syntaxe et la grammaire du texte de Collodi en déterritorialisant le langage pour y trouver une nouvelle diction. Le visage suit le même chemin que le corps dévasté. Bene apparaît de façon récurrente sur scène avec un visage galeux, rongé par un cancer qui fait partir sa peau en lambeaux. La chair est engagée dans un processus de pourriture. L'expérimentation des dysfonctionnements du corps, considérés dans ses fonctions les plus élémentaires, établit un lien évident avec le théâtre de la cruauté. La «machine actoriale» est une «marionnette-pantin», qui s'inscrit dans la longue ligne des acteurs-marionnettes évoqués par Kleist, Craig, Meyerhold, Artaud... Pinocchio est par excellence le pantin engagé dans un devenir-homme, tout en éprouvant une impossibilité d'être. Ce que

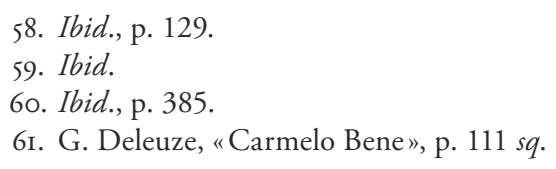


saisit la machine actoriale du pantin, c'est l'indécision d'un acteur qui oscille entre la marionnette et l'humain, ni sujet, ni objet. Les acteurs deviennent des forces, des flux jouant à nier toute forme de psychologie dramatique: un "super-acteur-machine " ${ }^{62}$. De cette manière, l'acteur ne suit pas les préceptes du théâtre naturaliste, qui lui demande d'imiter le réel, de répéter l'humain. Au contraire, le devenir-pantin de l'acteur permet une transformation, une variation. Être "machine actoriale», c'est alors être hors du soi, engagé dans un devenir-autre, dans une déterritorialisation vers d'autres corps et d'autres langages. La langue est travaillée par un devenir machine. Bene a recours à un soutien technique, sonorisant les acteurs, travaillant à une actorialité amplifiée, inventant une grammaire de voix qui tend à musicaliser la parole. Lacteur se saisit du texte et le modifie pour l'adapter à sa musique intérieure. L'acteur ne récite plus un texte appris par cœur: il le bouleverse, passe outre ses significations et met en relief ses fulgurances, ses vitesses: "Rien que des affects et pas de sujet, rien que des vitesses et pas de forme» résume Deleuze $^{63}$. La machine actoriale touche à plus d'abstraction, à mesure qu'elle est moins prise dans un drama, et plus impliquée dans un ergon, c'est-à-dire un «faire», un «travail». L'acteur n'est plus un interprète du drame, mais un acteur (au sens propre) de la machine théâtrale.

La suite naturelle de cet article serait une confrontation entre ces différentes hypothèses sur l'acteur de théâtre et les théories explorées par Gilles Deleuze dans l'Image-Mouvement et l'Image-Temps; ouvrages sur le cinéma qui regorgent de notes, remarques, indications concernant l'acteur de cinéma d'un nouveau type, comme les acteurs non-professionnels, les "acteurs-mediums", les acteurs de Robert Bresson, les acteurs burlesques, le cinéma de l'acteur-automate spirituel mais aussi les clowns, le fantôme et le travesti...

\section{Conclusion}

La visée de cet article est de nature prospective. L'essentiel pour nous, en tant que théoriciens, est de mettre ces propositions philosophiques sur le jeu d'acteur à l'épreuve de la scène de théâtre et sous le feu des questions

62. C. Bene, Autobiographie d'un portrait, p. 239.

63. G. Deleuze, Superpositions, p. 114. 
des élèves. Les pratiques pédagogiques existantes (Marceau, Dullin, Meyerhold) qui sont à la base des analogies faites ici, sont mobilisées à titre historique et documentaire, pas comme méthodes qui devraient absolument constituer le travail pratique.

Pédagogiquement, l'isolement des trois formes de jeu décrites par Gilles Deleuze est censé offrir aux élèves acteurs des repères pour s'orienter dans cette formulation théorique qui est ensuite destinée à être utilisée lors d'un travail à la table ou en situation, sur un plateau, lors d'un travail d'improvisation ou d'interprétation d'un texte de répertoire. Plutôt que prescrire des techniques de jeu, ce texte vise à susciter des interrogations, des essais non conventionnels vers des formes de jeu qui aident les élèves à sortir de leurs zones de confort ou de leurs habitudes de jeu.

C'est le travail que nous avons mené avec les élèves comédiens du Conservatoire régional de Toulouse en 2017-2018, en ayant à l'esprit de leur donner un vrai temps de "studio", comme l'entendait Meyerhold, à savoir un lieu et un espace de recherche improductifs, sans objectifs et sans spectateurs. À l'heure où en France la "recherche-création" est de plus en plus pratiquée, dans les écoles de théâtre comme à l'Université, l'un des enjeux de ces workshops a été de donner assez de confiance aux acteurs, en les confrontant à un matériau philosophique complexe, pour qu'ils et elles s'autorisent à puiser dans les textes des sciences humaines et sociales qui peuvent être des ressources insoupçonnées afin de questionner leurs pratiques et leurs méthodes de création. Voilà pourquoi ce texte se tient à poser des hypothèses qui ne demandent qu'à être pollinisées, fécondées ou rejetées par les praticiennes et praticiens avec leur savoir situé et vécu, car toute proposition théorique sur le théâtre ne saurait être validée autrement que par l'épreuve de la scène.

Flore Garcin-Marrou

Université Toulouse II Jean-Jaurès 


\section{BIBLIOGRAPHIE}

Artaud, Antonin, Euvres complètes, notées, 26 vols, Paris, Gallimard, 1956-1994.

—, Euvres, éd. par Évelyne Grossman, Paris, Gallimard, Quarto, 2004.

-, Pour en finir avec le jugement de dieu, in Euvres, éd. par Évelyne Grossman, Paris, Gallimard, Quarto, 2004, p. 1639-1654.

-, Van Gogh ou le suicidé de la société, in Euvres, éd. par Évelyne Grossman, Paris, Gallimard, Quarto, 2004, p. 1439-1463.

Artières, Philippe et al. (dir.), Le groupe d'information sur les prisons. Archives d'une lutte, 1970-1972, Paris, Éditions de l'IMEC, 2003.

Bene, Carmelo, Notre Dame des Turcs suivi de Autobiographie d'un portrait. Euvres complètes I, traduit de l'italien et préfacé par JeanPaul Manganara, Paris, POL, 2003.

Deleuze, Gilles, Nietzsche et la philosophie, Paris, Quadrige, 1997 (1962).

—, "La méthode de dramatisation", communication du 28/01/1967 à la Société Française de Philosophie, reprise dans le Bulletin de la Société Française de Philosophie, 61 (juillet-septembre 1967), p. 89-118, repris dans Deux régimes de fous et autres textes, Paris, Les Éditions de Minuit, 2003.

—, Différence et répétition, Paris, Épiméthée, 1996 (1968).

—, Logique du sens, Paris, Les Éditions de Minuit, coll. "Critique», 1969.

—, Superpositions (joint à Carmelo Bene, Richard III), Paris, Les Éditions de Minuit, 1979.

-, Francis Bacon. Logique de la sensation, Paris, L'Ordre philosophique, 2002 (1981).

—, "Carmelo Bene. L'histrion scélérat", in Jean-Pierre Leonardini, Marie Collin, Joséphine Markovits, Festival d'Automne à Paris 1972-1982, Paris, Temps Actuels, 1982.

—, Cinéma 2. L'image-temps, Paris, Les Éditions de Minuit, 1985.

—, Critique et clinique, Paris, Les Éditions de Minuit, 1993. 
—, Deux Régimes de fous. Textes et entretiens, 1975-1995, Paris, Les Éditions de Minuit 2003.

Deleuze, Gilles, Guattari, Félix, L’Anti-Edipe. Capitalisme et schizophrénie 1, Paris, Les Éditions de Minuit, 1972.

—, "Traité de nomadologie: la machine de guerre», in Mille plateaux. Capitalisme et schizophrénie 2, Paris, Les Éditions de Minuit, 1980, p. 434-527.

—, Qu'est-ce que la philosophie?, Paris, Les Éditions de Minuit, 1991.

Derrida, Jacques, "La double séance», in La dissémination, Paris, Seuil, 1972, p. 248-250.

GaY, Annie, «L'acteur délié. André Marcon dans Le Monologue d'Adramélech», Théâtrelpublic, 66 (1985), p. 13-14.

Green, Eugène, La parole baroque, Paris, Desclée de Brouwer, 2001.

Tournier, Michel, Célébrations, Paris, Mercure de France, 1999.

Mallarmé, Stéphane, Euvres complètes, 2 vols, présentation par Bertrand Maréchal, Paris, Gallimard, 2003,

Nietzsche, Friedrich, "Schopenhauer éducateur", in Considérations inactuelles III et $I V$, trad. H.-A. Baatsch, P. David, C. Heim, Ph. Lacoue-Labarthe et J.-L. Nancy, Paris, Gallimard, 1990, p. 15-96.

—, Par-delà bien et mal, traduction et présentation par Patrick Wotling, Paris, GF-Flammarion, 2000. 
